\title{
Estilos de aprendizaje en estudiantes universitarios y médicos residentes
}

\author{
R.A. Borraccia ${ }^{a, b, c}$, G. Guthman ${ }^{b}$, M. Rubio ${ }^{c}$, E.B. Arribalzaga ${ }^{a, c}$
}

Objetivos. Describir los estilos de aprendizaje a los que recurren los estudiantes de medicina al ingresar en la universidad, compararlos con los estilos al finalizar la carrera y en el posgrado inmediato, analizar la relación entre estilo de aprendizaje y desempeño académico, y estudiar la asociación entre estilos y la tendencia a elegir una especialidad al finalizar la carrera. Sujetos y métodos. Durante 2006 se incluyeron de forma prospectiva 102 estudiantes de medicina de segundo año (grupo pregrado inicio), 52 estudiantes del último año (grupo pregrado final) y 45 médicos de segundo año de residencia (grupo posgrado). Se determinó el estilo de aprendizaje con el cuestionario de Honey-Alonso. En la muestra pregrado final se incluyó la preferencia por una especialidad clínica o quirúrgica al finalizar la carrera, datos que fueron relacionados con los estilos preponderantes. Resultados. En pregrado inicio, la preferencia para adquirir información fue teórica en el $77 \%$ y el uso fue fundamentalmente reflexivo en el $86 \%$. En posgrado, la adquisición de información fue predominantemente teórica en el $67 \%$ y el uso reflexivo en el $70 \%$. La comparación demostró la disminución del modo reflexivo y el aumento del activo cuando se empleó la información al acercarse al posgrado $(p=0,069)$. En pregrado inicio hubo una tendencia al estilo 'asimilador' (70,6\%), mientras que el 'convergente' fue el más raro (6,9\%). Al comparar pregrado final y posgrado se demuestra en este último una reducción del estilo 'asimilador' ( $p=0,040$ ), a expensas del crecimiento 'convergente' $(p=0,034)$. Conclusiones. Los estudiantes tuvieron un estilo preponderantemente teórico y reflexivo (asimilador) al comenzar la carrera. Al finalizarla y en el posgrado disminuyó el estilo 'asimilador', en especial a expensas del crecimiento 'convergente'. No se pudo demostrar una relación entre los estilos de aprendizaje y el desempeño académico en anatomía. Al evaluar la presunta asociación entre estilo de aprendizaje y preferencia por una especialidad, se encontró que esta relación estaba más vinculada al efecto 'confundidor' del género; así, en las mujeres preponderó el estilo 'divergente' y la especialidad clínica, mientras que en los varones se observó un perfil 'asimilador'y la opción por una especialidad quirúrgica.

Palabras clave. Desempeño académico. Educación. Estilos de aprendizaje. Estudiantes. Medicina.

\section{Learning styles in university students and medical residents}

Aims. To identify the prevalent learning styles among students beginning Medical School and to compare these preferences with those found at the end of the carrier and during the residency program. The relationship between learning style and academic performance, as well as the association between learning preferences and postgraduate specialty selection were additionally studied. Subjects and methods. The HoneyAlonso learning style questionnaire was administered to 102 second-year students (pregraduate initial group), 52 lastyear students (pregraduate final group) and 45 physicians at a cardiology residency program (postgraduate group). Learning styles were compared within and between groups. The relationship between learning styles and academic performance in anatomy was assessed in the pregraduate initial group). The association between learning preferences and postgraduate specialty selection was studied in the pregraduate final group. Results. Learning preferences in the pregraduate initial group was theoretic (70\%) for acquiring information and reflexive (86\%) for using information respectively. In postgraduate group, preferences were theoretic (67\%) and reflexive (70\%), showing a marked decrease of the last style with respect to pregraduate initial ( $p=0.069$ ), at expense of an increase in active style. pregraduate initial showed a tendency towards 'assimilator' style (70.6\%), while

\footnotetext{
a Departamento de Bioestadística. Universidad Austral.

b Área de Investigación. Sociedad Argentina de Cardiología.

c Departamento de Cirugía. Facultad de Medicina. Universidad de Buenos Aires. Buenos Aires, Argentina.
}

Correspondencia Dr. Raúl A. Borracci. La Pampa $3030,1^{\circ}$. B. 1428 Buenos Aires, Argentina.

E-mail borracci@universia.com.ar 
'convergent' one was the rarest (6.9\%). When comparing pregraduate final to postgraduate group, a reduction of 'assimilator' style ( $p=0.040)$ and an increase of 'convergent' one ( $p=0.034)$ was observed. Conclusions. Students' learning preferences for acquiring and using information were theoretic and reflexive (assimilator) predominantly at the beginning of the carrier. At the end and at the postgraduate period, 'assimilator' style decreased at expense of an increase of the rest, especially at 'convergent' (theoretic and active) one. Although the 'assimilator' was the commoner, increase of 'convergent' style showed that at residency, individuals learn by solving problems and using knowledge in practice; this increase was done at expense of the active over the reflexive style. On the other hand, no relationship between learning style and academic performance in anatomy was found. Finally, an association between learning styles and postgraduate specialty selection showed that the last was more related to genre than to learning preferences.

Key words. Academic performance. Learning styles. Medicine. Students. Teaching.

\section{Introducción}

Las investigaciones en el área de la teoría del aprendizaje basada en la experiencia han demostrado que las personas aprenden de diversas formas [1]. Una de las variables más importantes que influyen en esta capacidad es el llamado 'estilo de aprendizaje, que indica la tendencia a desarrollar o elegir ciertas estrategias de aprendizaje sobre otras y que dependen fundamentalmente de la manera de adquirir la información y la forma de usarla. Los estilos de aprendizaje fueron descritos por Kolb [1], e incluyen dos formas opuestas de adquirir información, la conceptualización abstracta y la experiencia concreta, y dos maneras de usarla, la observación reflexiva y la experimentación activa. Por su parte, Honey, Munford y Alonso [2] han descrito las distintas características relacionadas con cada estilo de aprendizaje. Así, los estudiantes en los que predomina el estilo activo tienen preferencia por implicarse en nuevas experiencias y en el trabajo en equipo, mientras que en los de estilo reflexivo, tienden a observar y analizar la experiencia desde distintas perspectivas. Por su parte, el estilo abstracto o teórico se inclina por adaptar e integrar las observaciones dentro de teorías lógicas y complejas, y en el estilo concreto o pragmático hay tendencia a la aplicación prácti- ca de las ideas [3]. Existen algunos instrumentos validados, como los cuestionarios de Kolb [4] y de Honey-Alonso [5], capaces de discernir entre estilos de aprendizaje diferentes. El último de los cuestionarios consta de una evaluación anónima de 80 preguntas de respuesta dicotómica, que adjudica una puntuación para cada estilo, de acuerdo a la selección hecha por el alumno.

La investigación de los estilos de aprendizaje y los tipos psicológicos es actualmente objeto de estudio en muchas escuelas de medicina, dada su presunta relación con el desempeño académico, la elección de la especialidad y la satisfacción y eficiencia de los alumnos [3,6-14]. Además, el hecho de conocer el estilo de aprendizaje preponderante en un grupo podría servir para optimizar y potenciar los recursos que mejor incentiven el proceso de enseñanza. La mayoría de las investigaciones sobre estilos de aprendizaje en el nivel universitario destaca el perfil promedio del grupo estudiado, y la posibilidad de influir sobre el mismo, con miras a modificar la preponderancia de algún estilo en especial $[6-7,15]$. Por el contrario, existe poca información sobre la relación entre el estilo de aprendizaje y el desempeño durante la carrera de grado [3,12-13], así como tampoco se conoce la relación que pueda tener este perfil de aprendizaje con la tendencia a elegir una especialidad clínica o quirúrgica al finalizar los estudios, o las diferencias de estilo que pudieran darse entre estudiantes universitarios de los primeros años y aquéllos a punto de graduarse o recientemente graduados. En el caso del desempeño durante la carrera, podrían existir, fundamentalmente, resultados antagónicos si el estilo de aprendizaje fuera preponderantemente teórico y el de enseñanza o evaluación tuviera un perfil pragmático, o viceversa. De la misma forma, el aprendizaje de cada materia en particular podría requerir de algún estilo en especial. En el caso de la predilección o elección de una especialidad al finalizar la carrera, podrían existir requisitos diferentes para el buen desempeño en cada uno de ellas. Así, una especialidad clínica requeriría quizá de un perfil preponderantemente teórico-reflexivo, y una quirúrgica de un estilo más activo y pragmático.

De acuerdo con el marco teórico expuesto, los objetivos primarios de este estudio fueron describir los estilos de aprendizaje distintos a los que recurren los estudiantes de medicina en el momento de ingresar en la universidad, y comparar estos resultados con los estilos preponde- 


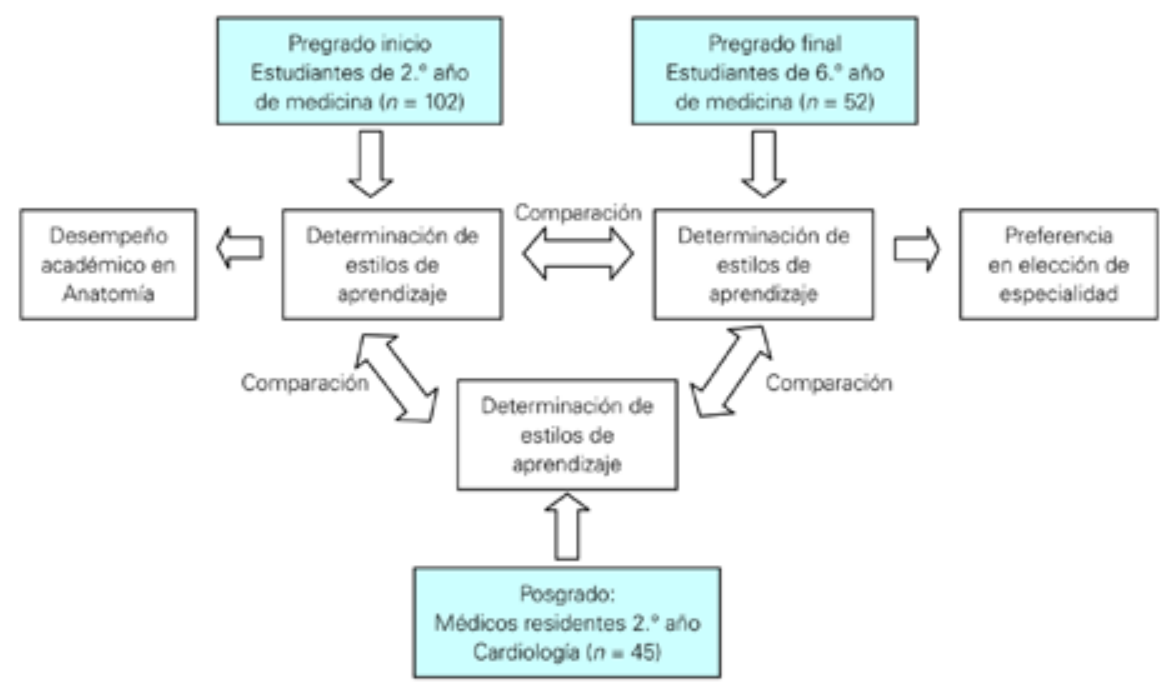

Figura 1. Diseño del estudio.

rantes al finalizar la carrera y en los primeros años del posgrado.

Por su parte, los objetivos secundarios fueron analizar la relación entre el estilo de aprendizaje de los estudiantes y su desempeño académico, y estudiar la asociación entre el estilo de aprendizaje de los estudiantes próximo a graduarse y la tendencia a elegir una especialidad clínica o quirúrgica al finalizar la carrera.

\section{Materiales y métodos}

Durante 2006 se estudiaron de forma prospectiva tres muestras de individuos con las siguientes características:

- Grupo pregrado inicio: 102 estudiantes de medicina al comienzo de su segundo año de la carrera.

- Grupo pregrado final: 52 estudiantes de medicina que cursan el último año de la carrera.

- Grupo posgrado: 45 médicos que comienzan su segundo año de la residencia de cardiología.

En todos los grupos se determinó el estilo de aprendizaje de acuerdo con la forma de adquirir y usar la información en base al cuestionario de Honey-Alonso [5]. Después de clasificar los dis- tintos estilos, se procedió a compararlos dentro del grupo y entre los grupos. Adicionalmente, en la muestra pregrado inicio se relacionaron los diferentes estilos de aprendizaje con el desempeño en la materia de anatomía (puntuación del examen final). Por su parte, en la muestra pregrado final se solicitó a los encuestados que señalaran la preferencia por una especialidad clínica o quirúrgica que debían elegir al finalizar la carrera. Este último dato fue relacionado posteriormente con los estilos preponderantes en el grupo. En la figura 1 se resume esquemáticamente el diseño del estudio.

\section{Determinación del estilo de aprendizaje}

La determinación de los distintos estilos de aprendizaje se realizó con la aplicación del cuestionario de Honey-Alonso [5]. La elección de este método se basó en la validación previa y en su condición de haber sido confeccionado originalmente en español [2,5,7]. Este cuestionario consta de una evaluación anónima de 80 preguntas de respuesta dicotómica que adjudica una puntuación para cada estilo de acuerdo con la elección hecha por el estudiante. La estandarización y representación sobre un eje cartesiano, o la diferencia neta entre cada par de estilos antagónicos permite ubicar cada individuo en un estilo preponderante. El cuestionario y la metodología para la aplicación de este método se detallan en el anexo. La validez 


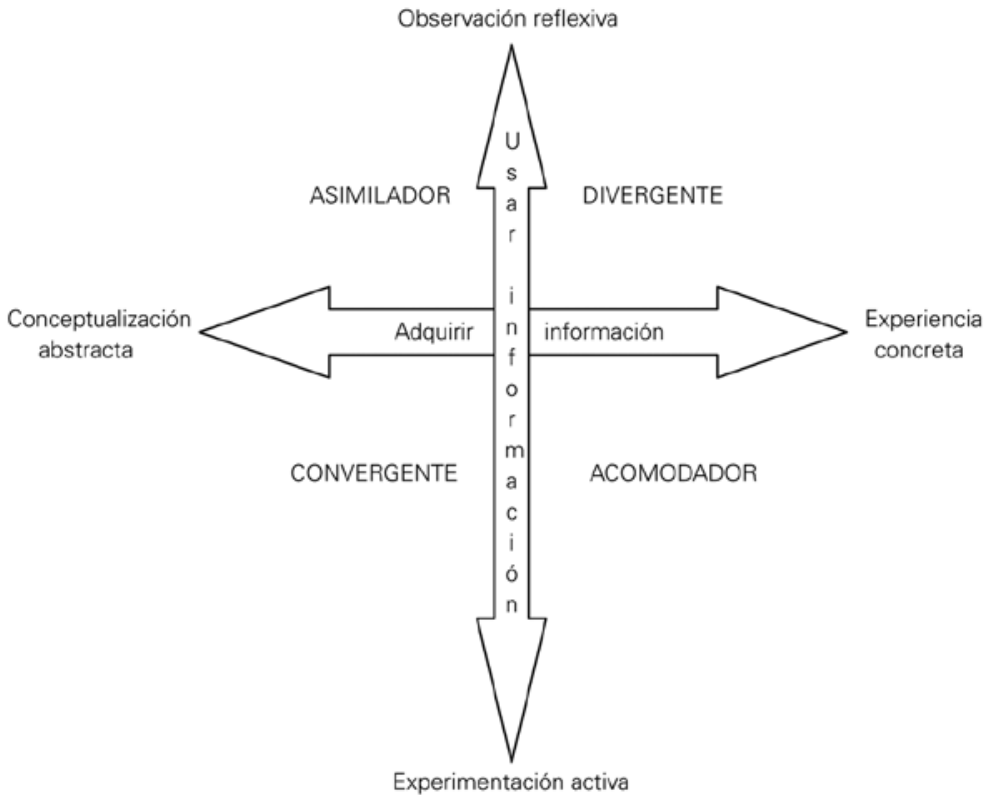

Figura 2. Estilos de aprendizaje y subtipos generados a partir de la combinación de la forma de adquirir y usar la información según Kolb [1].

local (fiabilidad) del modelo de Honey-Alonso se realizó con el test $\alpha$ de Cronbach [9].

El estilo preponderante de cada individuo se representó en sendos gráficos de dispersión. Los valores de $x$ e $y$ necesarios para su representación se obtuvieron con la diferencia neta entre las puntuaciones del cuestionario para cada par de estilos antagónicos (teórico frente a pragmático, y reflexivo frente a activo). La cuenta final del número de adjudicados a cada cuadrante determinó el porcentaje para cada estilo. La comparación de los porcentajes intragrupo y entre grupos se realizó con $\chi^{2}$ o test de probabilidad exacta de Fisher. El tamaño de la muestra se calculó considerando que los estilos teórico y reflexivo son los predominantes (aproximadamente $80 \%$ de los estudiantes), y la diferencia que había que detectar debía ser mayor del $30 \%$ en valor absoluto, con un nivel de confianza del $95 \%$ y un poder del $90 \%$.

\section{Definición y clasificación de los estilos de aprendizaje}

Para clasificar los estudiantes dentro de los subtipos de estilos de aprendizaje diferentes, se usó la división de Kolb [1], de acuerdo con el esquema de la figura 2. La definición de cada uno de estos subgrupos fue:

- Asimilador (abstracto-reflexivo): asimila gran cantidad de información. Tiende a aprender sistematizando la información en patrones y teorías unificadoras, sin interés particular en la aplicación práctica de los conocimientos. Reflexiona y luego actúa. Prefiere las clases a las actividades prácticas.

- Divergente (concreto-reflexivo): tiene facilidad para aprender de la experiencia después de analizarla desde perspectivas múltiples. Se interesa por los detalles concretos. No se interesa por los aspectos teóricos. Valora las relaciones interpersonales y prefiere trabajar en equipo.

- Acomodador (concreto-activo): tiene preferencia y facilidad para aprender con la experimentación o la práctica. No se interesa por los aspectos teóricos. Explora oportunidades y se arriesga. Prefiere las actividades prácticas a las clases.

- Convergente (abstracto-activo): aprende resolviendo problemas. Aplica el conocimiento a la práctica, a partir de la generación de modelos hipotéticos. Prefiere las actividades prácticas a las clases. 


\section{Determinación del rendimiento académico}

El rendimiento académico del grupo pregrado inicio se determinó con las notas finales de la materia Anatomía (Anatomía I y II) separados para cada estilo de aprendizaje. El anonimato entre las respuestas del cuestionario de estilos de aprendizaje y las notas de la materia se mantuvo con la adjudicación de un número de registro a cada estudiante. La comparación de los promedios y varianzas se realizó con ANOVA.

\section{Determinación de las preferencias por especialidad}

La relación entre los estilos de aprendizaje y las preferencias por elegir una especialidad clínica o quirúrgica en los estudiantes próximos a graduarse se determinó con la solicitud a los encuestados del grupo pregrado final que señalaran además la intención de elegir una u otra especialidad. Adicionalmente, los encuestados indicaron el sexo a fin de disponer de una variable potencialmente 'confundidora', habida cuenta de la tendencia de los varones a elegir especialidades quirúrgicas y de las mujeres a dedicarse a especialidades clínicas. La cuenta del número de adjudicados a cada cuadrante, separados por especialidad y sexo, determinó el porcentaje para cada estilo. La comparación de los porcentajes se realizó con $\chi^{2}$ o test de probabilidad exacta de Fisher.

\section{Limitaciones del diseño}

Una limitación que hay que tener en cuenta es la que se origina en un probable sesgo de inclusión en la muestra posgrado. Es este caso se han elegido sólo médicos que se hallaban en un programa de residencias clínicas (cardiología), en lugar de elegir otra especialidad, o médicos que no hubieran accedido a una residencia. Una muestra de posgrado que contemplase estas diferencias podría haber sido más representativa. Otra limitación del diseño es que por tratarse de un estudio transversal, los grupos comparados fueron independientes. De forma ideal, un mismo grupo seguido a través de los años podría haber mostrado la verdadera evolución temporal de los estilos de aprendizaje. Por último, el método de identificación de los estilos de aprendizaje usado en este trabajo estuvo basado en un cuestionario, procedimiento comúnmente conocido como 'autorreporte' (self-reports). Algunos estudios en alumnos de otras disciplinas han sugerido que los 'autorreportes' basados en cuestionarios podrían ser menos fiables que otro tipo de herramientas basadas en la observación directa del educando [11], lo que debiera tenerse en cuenta como otra limitación potencial de este estudio.

\section{Resultados}

A pesar de que el cuestionario aquí empleado fue validado previamente, se evaluó la validez local (fiabilidad) del modelo con el test $\alpha$ de Cronbach. Los valores encontrados para cada estilo fueron los siguientes: activo $(0,651)$, reflexivo $(0,512)$, teórico $(0,431)$ y concreto $(0,507)$.

En el caso de la muestra de pregrado inicio, la preferencia para la adquisición de la información fue predominantemente teórica (o abstracta) en el $77 \%(79 / 102)$ de los estudiantes frente a una predilección por la adquisición concreta (o pragmática) en el 23\% (23/102) restante. Para el caso del uso de la información, ésta fue de tendencia fundamentalmente reflexiva en el 86\% (88/102) de los individuos frente a un $14 \%(14 / 102)$ de propensión a la utilización activa de dicha información. En el otro extremo, al analizar la muestra de posgrado, se observó que la preferencia para la adquisición de la información fue predominantemente teórica (o abstracta) en el 67\% (30/45) de los médicos residentes frente a una predilección por la adquisición concreta (o pragmática) en el 33\% (15/45) restante. Para el caso del uso de la información, ésta fue de tendencia fundamentalmente reflexiva en el 70\% de los individuos frente a un 30\% de propensión a la utilización activa de dicha información. La comparación estadística que se observa en la figura 3 demuestra, en especial, la disminución porcentual del modo reflexivo y el consecuente aumento de la preferencia activa al usar la información a medida que se acerca al grupo de individuos del posgrado $(p=0,069)$. Los valores del grupo pregrado final también se muestran en esta figura: teórico, $71 \%$ (37/52); concreto, $29 \%$ (15/52); reflexivo, $85 \%$ (44/52), y activo, $15 \%$ (8/52).

De acuerdo con las preferencias en la adquisición y uso de la información combinadas se confeccionó la figura 4 , que muestra el diagrama de dispersión con la ubicación de cada estudiante (pregrado) y médico (posgrado) dentro de su es- 


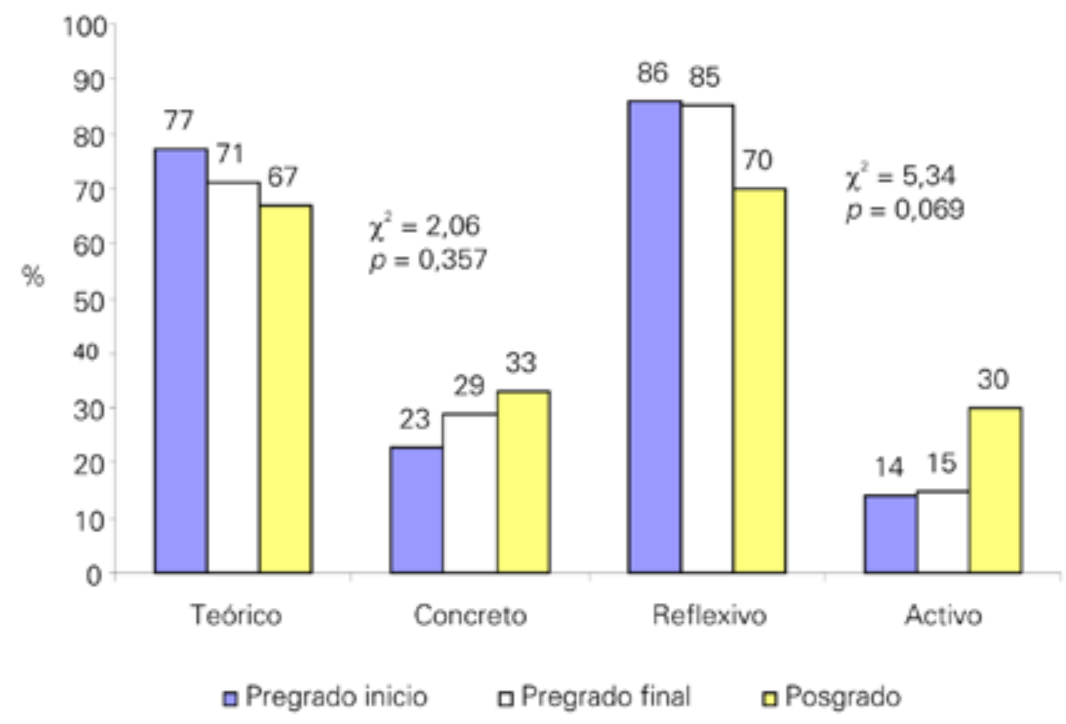

Figura 3. Comparación de las preferencias al adquirir y usar la información en los distintos grupos.

tilo de aprendizaje predominante. En el caso del pregrado inicio, la marcada tendencia a preferir el estilo asimilador indica que el 70,6\% (72/102) de los estudiantes tiende a aprender sistematizando la información en teorías unificadoras o patrones sobre los cuales reflexionar, y presta poco interés a sus aplicaciones prácticas. Por el contrario, el subtipo convergente, que es aquel que tiende a aplicar el conocimiento a la práctica, fue el más raro, con el $6,9 \%$ (7/102). Al comparar con el pregrado final y el posgrado, se demuestra en este último grupo una reducción significativa del estilo asimilador $(p=0,040)$, a expensas del crecimiento del resto de los patrones, en especial del convergente $(p=$ $0,034)$, lo que revela que los médicos residentes tienen una mayor preferencia por el estilo que tiende a aplicar el conocimiento a la práctica.

En el gráfico de área de la figura 5 se esquematiza el perfil de preferencias de aprendizaje del conjunto. Cada cuadrante representa el 100\% de predilección de dicho estilo; comparativamente, el área pintada indica la preferencia promedio por dicha forma de adquirir y usar la información.

$\mathrm{Al}$ analizar la relación entre el estilo de aprendizaje de los estudiantes del grupo pregrado inicio y su desempeño académico en la materia Anatomía, no se hallaron diferencias estadísticas, aunque se observó que aquellos individuos con estilo acomodador (concreto-activo) tuvieron mejores notas que el resto, mientras que el estilo convergente (abstracto-activo) fue el de peor desempeño. Las notas promedio de acuerdo al estilo fueron: asimilador, 6,80; divergente, 6,90; convergente, 6,00, y acomodador, 7,20 (ANOVA $F=0,634 ; p=0,59)$. La ausencia de peso estadístico en esta comparación probablemente se deba al bajo poder de la muestra para esta variable. De todas formas, debe destacarse que el estilo con mejor desempeño también depende de la forma de evaluación de la materia.

Por último, se analizó la asociación entre el estilo de aprendizaje de los estudiantes próximo a graduarse (grupo pregrado final) y la tendencia a elegir una especialidad clínica o quirúrgica al finalizar la carrera. La comparación de los distintos estilos en quienes eligieron una especialidad clínica o quirúrgica fueron respectivamente: asimilador, $66,7 \%$ frente a $55,6 \%, p=0,451$; divergente, $29,6 \%$ frente a $11,1 \%, p=0,272$; convergente, $0,0 \%$ frente a $11,1 \%, p=0,155$, y acomodador, $3,7 \%$ frente a $22,2 \%, p=0,146$. A pesar de que tampoco se encontraron diferencias estadísticas entre los estilos y la opción por una u otra especialidad, existió una clara tendencia hacia los estilos convergente $y$ acomodador entre quienes optaron por una especialidad quirúrgica frente a una preponderancia del estilo divergente entre quienes eligieron una especialidad clínica. La falta de una significación 
a

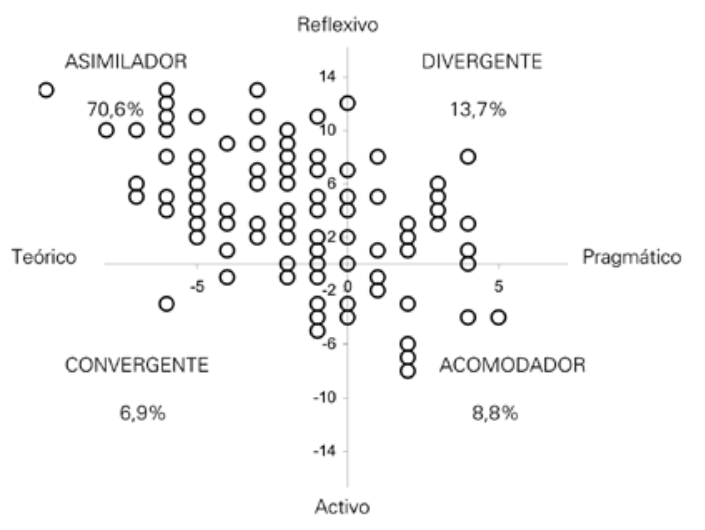

b

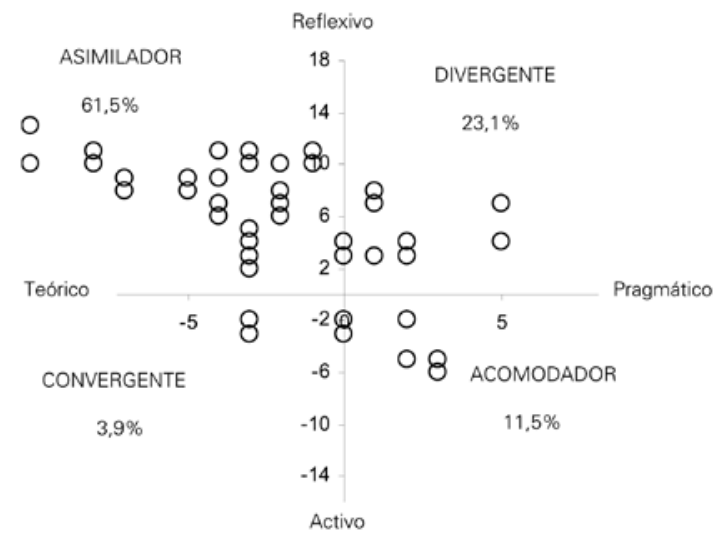

C

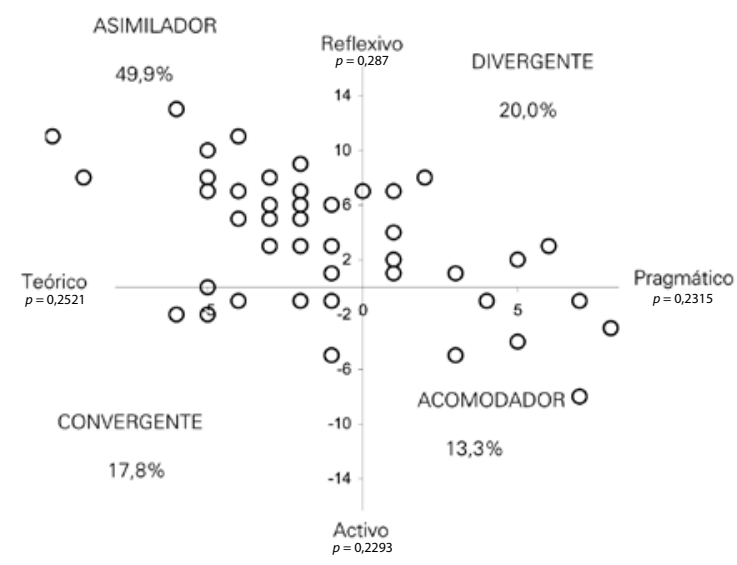

Figura 4. Diagrama de dispersión con el estilo preponderante de cada estudiante -pregrado inicio (a) y pregrado final (b)- y médico -posgrado (c)-. Los porcentajes señalan la proporción de individuos en cada cuadrante (perfil de preferencias individuales). Los valores comparativos de $p$ que se señalan en el gráfico de posgrado muestran las diferencias encontradas entre este grupo y los de pregrado inicio y pregrado final.

estadística en el $5 \%$ podría deberse una vez más a la falta de potencia de la muestra. Una de las hipótesis planteadas sobre este análisis se refería a la posibilidad de la existencia de alguna variable con efecto 'confundidor' que pudiera alterar la interpretación de los resultados. Por ello se analizó en este mismo grupo las preferencias por cada estilo de aprendizaje de acuerdo con el sexo. Este análisis reveló que el 76,9\% (20/26) de las mujeres prefirió una especialidad clínica, mientras que el $60 \%(12 / 20)$ de los varones optaron por una quirúrgica $(p=$ $0,01)$. En particular, las mujeres tuvieron preponderantemente preferencia por el estilo divergente (el mismo de las especialidades clínicas), mientras que los varones presentaron una distribución de preferencias de estilos muy similar a la observada entre quienes eligieron especialidades quirúrgicas (asimilador, $42,9 \%$ frente a $83,3 \%, p=0,007$; divergente, $42,9 \%$ frente a $0,0 \%, p=0,0009$; convergente, $7,1 \%$ frente a $0,0 \%, p=0,493$, y acomodador, $7,1 \%$ frente a $16,7 \%, p=0,396$, para las mujeres y varones respectivamente). Por lo tanto, es muy probable que las relaciones observadas entre estilos de aprendizaje y elección de la especialidad estén más relacionadas con el género (efecto 'confundidor').

\section{Discusión}

En este estudio se demostró que los estudiantes universitarios evaluados en esta cohorte tienen 


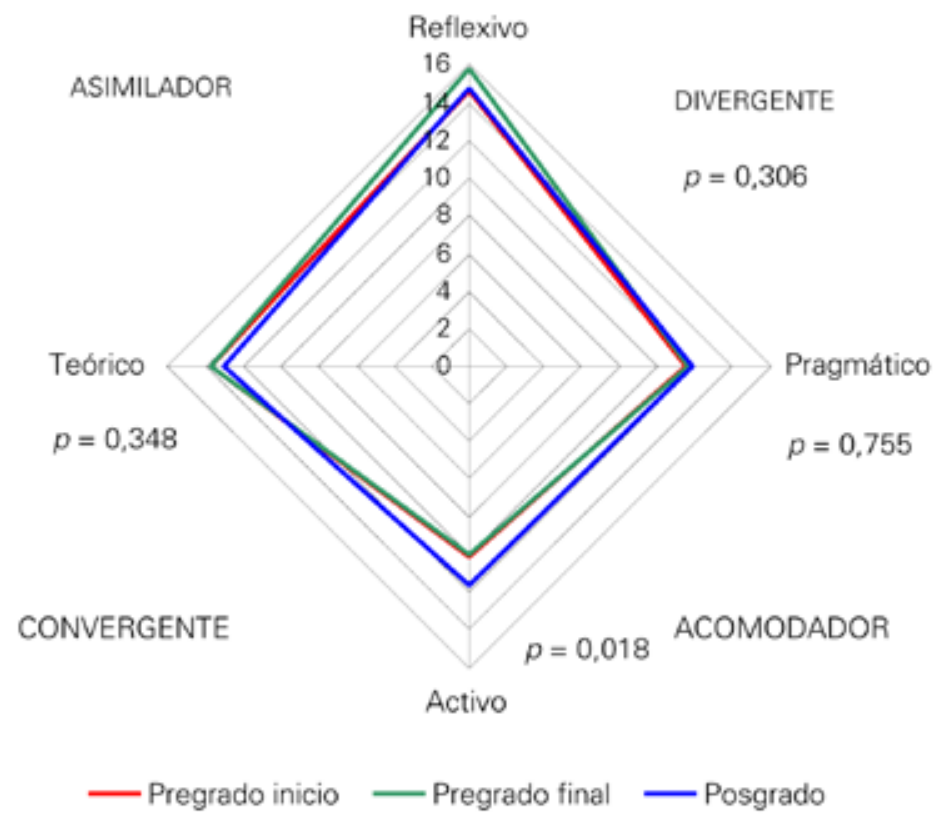

Figura 5. Puntuaciones promedio obtenidas para cada estilo de aprendizaje en la muestra de pregrado inicio, pregrado final y posgrado. Los valores comparativos de $p$ se señalan al lado de cada estilo (ANOVA de un factor).

un estilo preponderantemente teórico y reflexivo (asimilador) cuando se analiza la forma de adquirir y usar la información al comienzo de la carrera. Al comparar estos resultados con los obtenidos en estudiantes avanzados y en médicos recientemente graduados, se observó una tendencia a la disminución del estilo asimilador a expensas del crecimiento del resto de los patrones, en especial del convergente (teórico y activo). Aunque el modelo asimilador continuó siendo el preponderante, el crecimiento del estilo convergente señala que, en la residencia, los individuos tienden a aprender con la resolución de problemas y la aplicación del conocimiento en la práctica a partir de la generación de modelos hipotéticos. Como se observa en la figura 5, el incremento del estilo convergente en el grupo de graduados se realizó a expensas del crecimiento de las puntuaciones asociadas al patrón activo sobre el reflexivo. Esto indica que los individuos se volcaron más hacia la experimentación activa, la cual promueve la disposición a actuar y tomar decisiones, como ocurre en los programas de residencia. Es de destacar que esta preferencia por el estilo activo no se observó al finalizar la carrera, sino en el posgrado, lo que indicaría que el cambio de actitud no ocurre durante la carrera de grado.

En un grupo de 279 estudiantes ingresados a la Escuela de Medicina de la Universidad Católica de Chile entre 2000 y 2002, se encontró que la distribución de los estilos de aprendizaje fue asimilador en el $54 \%$ de los alumnos, convergente en el $23 \%$, divergente en el $14 \%$ y acomodador en el 9\% [6]. Estas proporciones son parecidas a las reportadas en nuestro estudio, a excepción del estilo convergente, que en la muestra estudiada fue muy inferior a la comunicada por estos autores (pregrado inicio $=6,9 \%$ ). Por otro lado, en un estudio retrospectivo sobre una cohorte de 66 estudiantes del último año de la carrera de medicina, Bitran et al [3] observaron que el estilo asimilador fue el más frecuente (44\%), seguido por el convergente (35\%), el divergente (11\%) y el acomodador (10\%). Estos datos contrastan con nuestros hallazgos en el grupo pregrado final, en el cual los aprendices reflexivos (asimiladores + divergentes) constituyen el 84,6\% de los casos. En la muestra posgrado se obtuvo un crecimiento del estilo convergente a expensas de los dos estilos previamente mencionados. De 
forma característica, el grupo pregrado final tuvo una proporción mayor del estilo divergente que el comunicado en el trabajo de Bitran et al [3]. Una razón que podría explicar esta diferencia es la alta proporción de mujeres en nuestra muestra (54\%), diferente a la del trabajo comparado (39\%). Como fuera observado en el grupo pregrado final, las mujeres tuvieron un perfil predominantemente asimilador + divergente frente a un estilo más asimilador puro en los varones.

Por otra parte, en el trabajo aquí presentado no se pudo demostrar una relación entre los estilos de aprendizaje de los estudiantes y el desempeño en la materia Anatomía. Si bien se observó una tendencia que desfavoreció al estilo convergente, ésta no pudo corroborarse desde el punto de vista estadístico. Esta situación pudo deberse tanto a una baja potencia de la muestra, como a las condiciones especiales de la enseñanza y evaluación de la materia que podrían considerar estilos antagónicos (enseñanza teórica frente a evaluación práctica). Por otra parte, estudios previos ya han señalado que los estudiantes podrían adaptar sus estrategias de aprendizaje a las demandas percibidas en cada materia [16].

En estudios de hace una década, algunos autores han encontrado una asociación entre los estilos de aprendizaje y el rendimiento académico medido a través de las notas de alguna asignatura específica [17-19]. En nuestro caso no fue posible hallar una relación entre los estilos y el rendimiento en la materia Anatomía, posiblemente debido a la baja potencia de la muestra. De la misma forma, otros autores tampoco encontraron una asociación entre los estilos y el rendimiento basado en las notas promedio de toda la carrera [3]. En un estudio de Lynch et al. [12], que evaluó el desempeño de 227 estudiantes de tercer año en el examen USMLE (United Status Medical Licensing Examination, step 1), se halló una correlación entre el estilo en que preponderaba la conceptualización abstracta (asimilador + convergente) y los resultados de dicho examen. Aunque la asociación alcanzó el valor de significación de 0,05 , los coeficientes de correlación fueron algo pobres $(r=0,20-0,33)$, lo que pone en duda la validez de estos resultados. Otros estudios que encontraron una relación entre los estilos de aprendizaje y el desempeño en los exámenes finales [13] no usaron la clasificación basada en las preferencias de adquisición y uso de la información, sino un modelo basado en la escala de aprendizaje superficial, profundo y estratégico según Newble [14].

Por último, al analizar una presunta asociación entre el estilo de aprendizaje de los estudiantes próximo a graduarse y la preferencia a elegir una especialidad clínica o quirúrgica, se encontró que esta relación estaba más relacionada con el efecto 'confundidor' del género que con los propios estilos. El análisis de estilos y preferencias de acuerdo con el sexo reveló que la mayoría de las mujeres prefirió una especialidad clínica, mientras que más de la mitad de los varones optó por una quirúrgica. Asimismo, las mujeres tuvieron preponderantemente preferencia por el estilo divergente (el mismo de las especialidades clínicas), mientras que los varones presentaron una distribución de preferencias de estilos muy similar a la observada entre quienes eligieron especialidades quirúrgicas.

El enfoque sobre los estilos de aprendizaje abordado en nuestro estudio es únicamente una forma de analizar los perfiles o las preferencias de aprendizaje de los estudiantes. Como ya fue mencionado, otros trabajos utilizan la clasificación en estilos superficial, profundo y estratégico [14]. En particular, algunos estudios han encontrado que el estilo de aprendizaje llamado superficial es el más común entre quienes ingresan en las escuelas de medicina de Canadá [20]. Otros trabajos dividen los estilos en independiente, dependiente, colaborador, evitador, facilitador y competitivo $[21,22]$. Por último, algunos autores individualizan los estilos de aprendizaje basándose en la preferencia por distintas modalidades preceptuales de adquirir la información, como la visual, la auditiva, la táctil y la cinestésica $[23,24]$.

Dado que la muestra posgrado correspondió a médicos que se hallaban en un programa de residencias clínicas (cardiología), la interpretación de que los estilos de aprendizaje evolucionan hacia un aumento del patrón convergente y a una disminución del asimilador, podría reinterpretarse como que los médicos residentes tienen un mayor patrón convergente, pues éste sería el más adecuado para ser elegible para un programa de residencia. Como consecuencia, aquellos que no pudieron acceder a una residencia tendrían un patrón más asimilador, como el encontrado en el pregrado. Esta hipótesis sólo podría aclararse en estudios futuros que comparasen muestras de posgrado con médicos que hayan accedido o no a un programa de residencia. 
En conclusión, en este estudio se demostró que los estudiantes de medicina tienen un estilo preponderantemente teórico y reflexivo (asimilador) para adquirir y usar la información al comenzar la carrera. Al comparar estos resultados con los obtenidos en estudiantes que finalizaban la carrera y en médicos recientemente graduados, se observó una tendencia a la disminución del estilo asimilador a expensas del crecimiento del resto de los patrones, en especial del convergente (teórico y activo); este último incremento se realizó a expensas del crecimiento del patrón activo sobre el reflexivo. Destaca que esta preferencia por el estilo activo no se observó al finalizar la carrera, sino en el posgrado. Por otra parte, no se pudo demostrar una relación entre los estilos de aprendizaje de los estudiantes y el desempeño académico en la materia Anatomía. Por último, al evaluar la presunta asociación entre el estilo de aprendizaje de los estudiantes próximo a graduarse y la preferencia a elegir una especialidad clínica o quirúrgica, se encontró que esta relación estaba más vinculada con el efecto confundidor del género que con los propios estilos. El análisis de perfiles y preferencias de acuerdo con el sexo reveló que en la mayoría de las mujeres preponderó el estilo divergente y la preferencia por una especialidad clínica, mientras que en más de la mitad de los varones se observó un perfil asimilador y la opción por una especialidad quirúrgica.

\section{Bibliografía}

1. Kolb D. Experiential learning: experience as the source of learning and development. New Jersey: Prentice Hall, PTR; 1984.

2. Alonso C, Gallego D, Honey P. Los estilos de aprendizaje. Procedimientos de diagnóstico y mejora. Bilbao: Mensajero; 1994.

3. Bitran $M$, Lafuente $M$, Zúñiga $D$, Viviani $P$, Mena $B$. ¿Influyen las características psicológicas y los estilos de aprendizaje en el rendimiento académico de los estudiantes de medicina? Un estudio retrospectivo. Rev Med Chile 2004; 132: 1127-36.

4. Kolb D. Inventario de los estilos de aprendizaje: Inventario autoevaluativo y su interpretación. Boston: TRG Hay/McBer; 1981.

5. Alonso C. Análisis y diagnóstico de los estilos de aprendizaje en estudiantes universitarios. Madrid: Universidad Complutense; 1992.

6. Bitran M, Zúñiga D, Lafuente M, Viviani P, Mena B. Tipos psicológicos y estilos de aprendizaje de los estudian- tes que ingresan en medicina en la Pontificia Universidad Católica de Chile. Rev Med Chile 2003; 131: 1067-78.

7. Canaleja-Pérez MC, Martínez-Martín ML, Pineda-Ginés MC, Vera-Cortés ML, Soto-González M, Martín-Marino $\mathrm{A}$, et al. Estilos de aprendizaje en los estudiantes de enfermería. Educ Med 2005; 8: 83-90.

8. Luján HL, Di Carlo SE. First-year medical students prefer multiple learning styles. Adv Physiol Educ 2006; 30: 13-6.

9. Cook DA. Reliability and validity of scores from the index of learning styles. Acad Med 2005; 80: S97-101.

10. Armstrong E, Parsa-Parsi R. How can physicians' learning styles drive educational planning? Acad Med 2005; 80: 680-4.

11. Veenman MV, Prins FJ, Verheij J. Learning styles: selfreports versus thinking-aloud measures. Br J Educ Psychol 2003; 73: 357-72.

12. Lynch TG, Woelfl NN, Steele DJ, Hanssen CS. Learning style influences student examination performance. Am J Surg 1998; 176: 62-6.

13. McManus IC, Richards P, Winder BC, Sproston KA. Clinical experience, performance in final examinations, and learning style in medical students: prospective study. BMJ 1998; 316: 345-50.

14. Newble D, Entwistle N. Learning styles and approaches: implications for medical education. Med Educ 1986; 20: 162-75.

15. Linares AZ. Learning styles of students and faculty in selected health care professions. J Nurs Educ 1999; 38: 407-14.

16. Ramsden P. Student learning and perceptions of the academic environment. High Educ 1979; 8: 411-28.

17. Newland J, Woelfl NN. Learning style and academic performance within a group of sophomore Medical students. Acad Med 1992; 67: 349-52.

18. Davies S, Rutledge C, Davies T. Students' learning styles do affect performance. Acad Med 1995; 70: 659-60.

19. Sobral D. Diagnostic ability of medical students in relation to their learning characteristics and preclinical background. Med Educ 1995; 29: 278-82.

20. Aaron S, Skakun E. Correlation of students' characteristics with their learning styles as they begin Medical School. Acad Med 1999; 74: 260-2.

21. Grasha A. Teaching with style. A practical guide to enhancing learning by understanding and teaching styles. New York: Alliance Publishers; 1996.

22. Vaughn L, Baker R. Teaching in the medical setting: balancing teaching styles, learning styles and teaching methods. Med Teacher 2001; 23: 610-2.

23. Morse JS, Oberer J, Dobbins JA, Mitchell D. Understanding learning styles: implications for staff development educators. J Nurs Staff Dev 1998; 14: 41-6.

24. Curry L. Cognitive and learning styles in medical education. Acad Med 1999; 74: 409-13. 